\title{
Behavioural Lifestyle Intervention Study (BLIS) in patients with type 2 diabetes in the United Arab Emirates: A randomized controlled trial
}

\author{
Sarah Abdi $^{{ }^{*}}$, Amena Sadiya $^{1}$, Samia Ali ${ }^{2}$, Shiby Varghese ${ }^{2}$ and Salah Abusnana ${ }^{2}$
}

\begin{abstract}
Background: Lifestyle modification is a cornerstone of the management of type 2 diabetes. However, in the United Arab Emirates, a country where type 2 diabetes is highly prevalent, non-compliance with a healthy lifestyle has been reported in many diabetic Emirati patients. The use of behavioural theories in lifestyle counselling is believed to facilitate behavioural changes, nevertheless, there are no published data regarding the use of structured behavioural lifestyle programmes tailored to suit Emirati culture. The primary objective of this study was to develop a behavioural lifestyle programme and to evaluate its effectiveness in improving glycaemic control in Emirati patients with type 2 diabetes.

Methods: The Behavioural Lifestyle Intervention Study (BLIS) was a translational randomized controlled trial in which patients $(n=35)$ were randomly assigned to the intervention or control group. Patients in the intervention group went through a six-month behavioural lifestyle programme composed of 8 sessions, whereas patients in the control group received standard care. Cognitive behavioural theory was the underpinning theory for the lifestyle intervention. $\mathrm{HbA} 1 \mathrm{c}$ levels were the trial's primary outcome measure, and the main dietary factor targeted for change was carbohydrate intake. They were measured at baseline, 3 months and 6 months and were assessed using one-way ANOVA at a significance level of $P<0.05$. All of the patients were then followed up at 1 year on all outcome measures.
\end{abstract}

Results: At 6 months, the HbA1c levels of the patients $(n=18)$ in the intervention group were significantly reduced $(-1.56 \pm 1.81, P<0.05)$, whereas no significant change was observed in the patients of the control group. Similarly, both carbohydrate intake from cereals and total carbohydrate intake (in grams) were reduced $(p<0.05)$ in the intervention group, by $32.92 \pm 54.34$ and $20.94 \pm 56.73$, respectively. At 1 year, the patients in the intervention group maintained a significant reduction in HbA1c levels $(-1.12 \pm 1.46, \mathrm{p}<0.05)$, whereas no change was observed in the control group.

Conclusion: The behavioural lifestyle intervention programme was effective in improving glycaemic control and compliance with carbohydrate intake goals in Emirati patients with type 2 diabetes. Larger randomized controlled trials are needed to validate these results and to identify key behavioural strategies that will improve compliance to lifestyle modifications in real life.

Trial registration: Clinicaltrials.gov trial identifier NCT02386930

Keywords: UAE, Lifestyle, Cognitive behavioural theory, type 2 diabetes

\footnotetext{
* Correspondence: sarah.abdi@rcdr.ae

'Clinical Dietitian, Rashid Center for Diabetes and Research, Ministry of Health, P.O BOX 21499, Ajman, United Arab Emirates

Full list of author information is available at the end of the article
}

(c) 2015 Abdi et al. Open Access This article is distributed under the terms of the Creative Commons Attribution 4.0 International License (http://creativecommons.org/licenses/by/4.0/), which permits unrestricted use, distribution, and reproduction in any medium, provided you give appropriate credit to the original author(s) and the source, provide a link to the Creative Commons license, and indicate if changes were made. The Creative Commons Public Domain Dedication waiver (http://creativecommons.org/publicdomain/zero/1.0/) applies to the data made available in this article, unless otherwise stated. 


\section{Background}

Type 2 diabetes is a serious growing public health burden in the United Arab Emirates (UAE) and is considered a major contributor to mortality and morbidity rates [2]. The International Diabetes Federation (IDF) had estimated 803,900 people living with diabetes in UAE in 2014 and reported it one of the highest prevalence in the Middle East and North Africa region (MENA) [1], highlighting the need for interventions to manage diabetes, prevent its complications and reduce its associated economic burden [3].

In overweight and obese patients, lifestyle modifications, including nutrition therapy, regular physical activity and weight loss, are considered fundamental for the management of type 2 diabetes [4]. However, compliance with healthy lifestyle practices in real life has been found to be a great challenge in Emirati patients with type 2 diabetes $[5,6]$. The use of behavioural theories as a theoretical framework for lifestyle counselling is thought to facilitate behavioural changes [7]. A commonly used theory in nutrition counselling is cognitive behavioural theory (CBT) which assumes that behaviours are acquired and can be modified through different strategies [7]. Examples of these strategies include goal setting, self-monitoring, problem solving, stimulus control, which help patients analyse and act on external and internal cues to unhealthy dietary patterns and a sedentary lifestyle [7].

Large CBT lifestyle trials have shown significant results for both the prevention of diabetes, such as the Diabetes Prevention Program (DPP) [8], and the management of its outcomes, such as the Look Ahead trial [9]. The significant outcomes of these studies were said to have the potential to direct strategies for lifestyle management of obesity and type 2 diabetes [10]. Researchers then aimed to investigate the reproducibility of these interventions in real-world practice, which contrary to large controlled trials, is subject to several limitations such as limited resources and insufficient time.

Some of the studies aimed at translating these interventions into real-world settings yielded promising results, particularly in reducing the incidence of type 2 diabetes and its risk factors [11-17]. However, a limited number of studies of translational behavioural lifestyle interventions for patients with type 2 diabetes have been conducted and have led to mixed results [18-20]. Two translational randomized controlled trials (RCTs) reported significant improvement in glycaemic control in patients with type 2 diabetes six months after a lifestyle intervention programme $[18,19]$, whereas Welchen et al. [20] did not show a clinically meaningful improvement in HbA1c at 6 and 12 months after a cognitive behavioural lifestyle treatment. Moreover, these 3 trials were conducted in Western and Asian populations, and reports of the impact of behavioural lifestyle programmes on outcomes in type 2 diabetes patients in the Arab Gulf region are scarce. Replicating these programmes in Arab populations without taking into consideration regional and cultural variability in dietary and physical activity patterns might be inappropriate. Hence, our primary aim was to develop a structured behavioural lifestyle programme tailored to suit Emirati dietary and physical activity habits. We conducted the programme for 6 months, and the patients were also followed-up at 1 year. We evaluated its effectiveness in improving HbA1c levels in patients with type 2 diabetes as compared to standard practices. Our secondary aim was to evaluate its effectiveness in improving associated outcomes such as blood pressure, lipid profiles, weight and compliance with dietary and physical activity goals.

\section{Methods}

\section{Study design}

The Behavioural Lifestyle Intervention Study (BLIS) was a six-month randomized controlled trial with two parallel arms. Randomization occurred using computergenerated random number tables after assessments for inclusion/exclusion criteria. The patients were assigned to the intervention or control arm and were matched for gender. The patients in the intervention group then received a 6-month lifestyle programme, while the patients in the control group received standard care. Upon completion of the intervention, all of the patients were followed up at 1 year.

\section{Setting and participants}

The study was conducted at Rashid Centre for Diabetes and Research (RCDR), a tertiary care centre for diabetes in Ajman, UAE. Patients were referred to RCDR from primary health care centres in the Northern Emirates for specialized diabetes care. As part of the multidisciplinary care approach at RCRD, all of the patients were scheduled to meet a clinical dietitian 1-2 weeks after their initial consultation with the endocrinologist. All patients scheduled for the dietitian's visit were assessed for the eligibility criteria. If eligible, patients were then informed about the study during their visit to the lifestyle clinic by the research personnel. To participate in the trial, the patients were required to meet the following inclusion criteria: a) 18-60 years old; b) clinical diagnosis of type 2 diabetes; c) HbA1c $\geq 7 \%$; d) body mass index (BMI) $\geq$ 25 (weight $(\mathrm{Kg}) /$ height $\left(\mathrm{m}^{2}\right)$ ); e) absence of a major physical disability that would restrict participation in moderate physical activity; f) absence of a serious heart condition, such as heart failure, heart attack, or stroke within the last 3 months; g) absence of proliferative retinopathy or kidney failure; and $\mathrm{h}$ ) absence of current participation in a weight management or lifestyle programme. Of the 55 patients who were potentially eligible for the trial, $31 \%(n=17)$ 
were not eligible and $1 \%(n=3)$ refused to participate. Of the 35 randomized patients, $83 \%$ completed the trial $(n=29)$ (Fig. 1). All patients were recruited between September and December 2013.

\section{Intervention}

All of the patients in the intervention group underwent a six-month lifestyle programme composed of 8 sessions, 4 individual sessions and 4 telephone calls, which were delivered by clinical dietitians (Fig. 2). CBT was the underpinning theory used to facilitate behavioural change. Evidence-based medical nutrition therapy was followed with all of the patients; however, the counselling method used in the intervention group was adapted to the main CBT strategies i.e., goal setting and self-monitoring. Goal setting was implemented by providing the patients with a goal chart at their baseline visit. This chart included SMART (smart, measurable, achievable, relevant and timed) goals for dietary changes, weight loss and physical activity. Carbohydrate intake was the main dietary factor targeted for change because the monitoring of carbohydrate intake is considered a key strategy in achieving glycaemic control [21]. Additionally, reducing carbohydrates intake is arguably more relevant to the Emirati community than reducing fat intake observed in other lifestyle interventions [10]. This is because, as observed in clinical practice, the Emirati diet is rich in carbohydrates sources such as rice, dates and juices. The weight loss targets were individualized based on the patients' weight loss history and readiness for weight loss; these targets ranged from 5-7 \%. The patients were provided with calorie-based diets (1200-1800 kcal) to help them achieve their weight loss targets. Physical activity targets based on the patients' current levels of exercise were also provided. These targets were set so that the patients would achieve a minimum of 30 minutes of moderate physical activity 5 times per week by the end of the trial. During each individual session, the patient's goal charts were reviewed, and new goals were set. Self-monitoring was encouraged through the maintenance of a diet diary, which was developed and provided at the initial assessment. In addition to individual sessions, the patients received 4 telephone calls to their mobile phones throughout the six-month trial. The telephone calls were conducted in accordance with a structured topic guide and included follow-up on the individual SMART goals, reinforcement of the use of the diet diaries, questions regarding the regularity of physical exercise, and the suggestion of solutions for problems encountered as

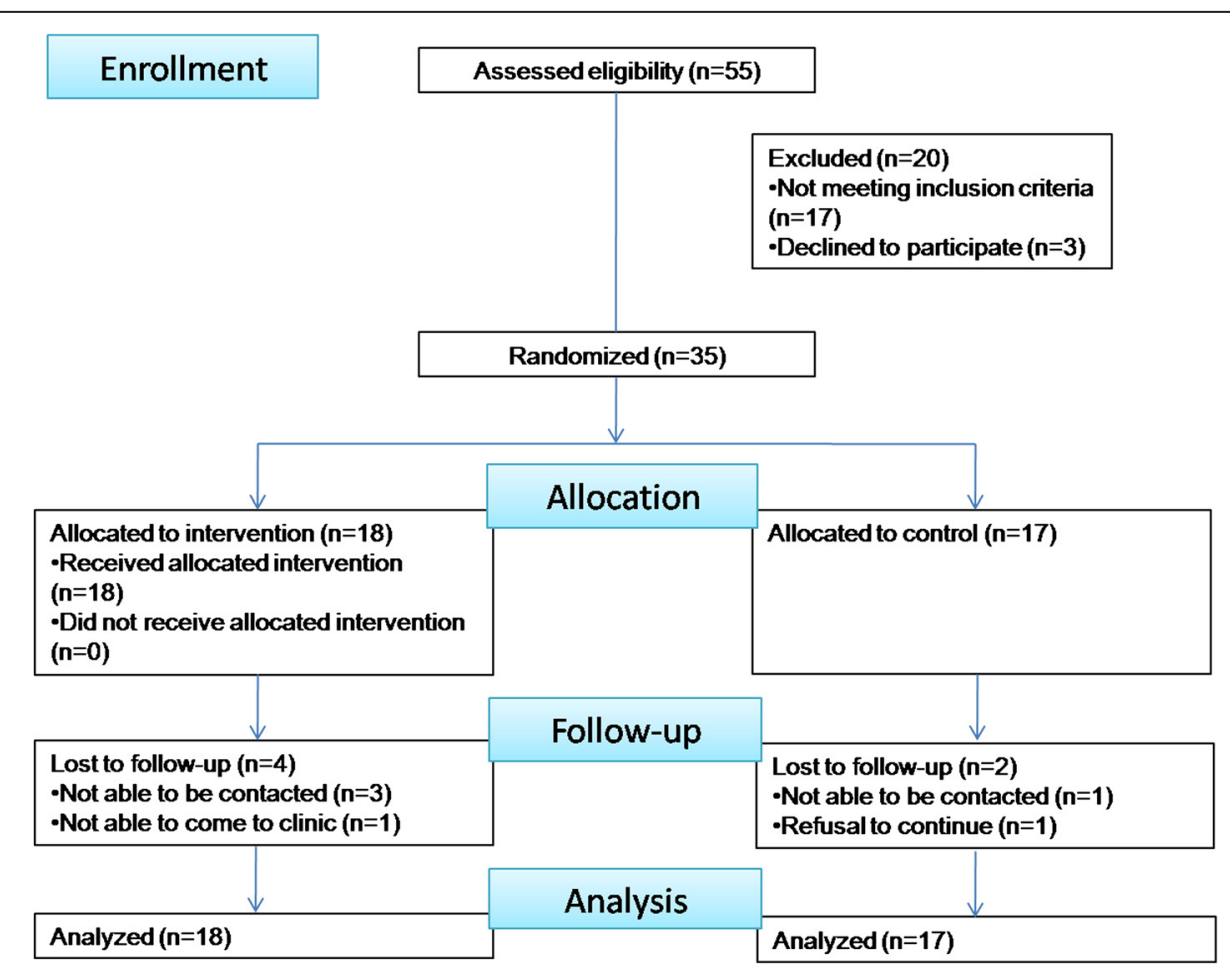

Fig. 1 Flow diagram of subjects through the different phases of the randomized controlled trial. Computer-generated random number tables were used for randomization after the patients were assessed with respect to the inclusion/exclusion criteria. The patients were then allocated to the intervention or control arm and were matched for gender. The patients in the intervention group then proceeded to receive a 6-month lifestyle programme, and the patients in the control group received standard care. Of the 55 patients who were potentially eligible for the trial, $31 \%(n=17)$ were not eligible, and $1 \%(n=3)$ refused to participate. Of the 35 randomized patients, $83 \%$ completed the trial $(n=29)$, with an attrition rate of $17 \%$. The data analysis was performed in accordance with the intention-to-treat analysis protocol 


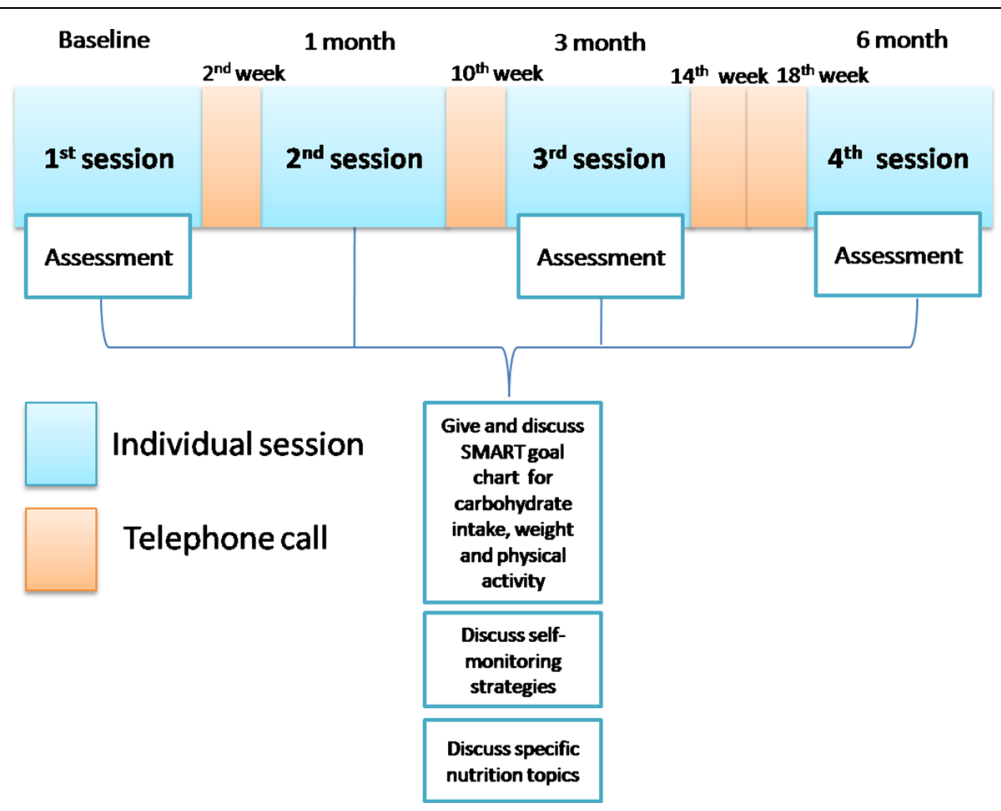

Fig. 2 Design of the behavioural lifestyle intervention study (BLIS). All of the patients in the intervention group underwent a six-month lifestyle programme composed of 8 sessions, 4 individual sessions and 4 telephone calls, which were administered by clinical dietitians. Cognitive behavioural theory was the underpinning theory used to facilitate behavioural changes. Accordingly, strategies such as self-monitoring and goal setting were used to improve compliance with healthy lifestyle practices. Goal setting was implemented by providing the patients with a goal chart at the baseline visit. These charts included SMART (smart, measurable, achievable, relevant and timed) goals for carbohydrate intake, weight loss and physical activity. During each individual session, the goal charts were reviewed and new goals were set. Self-monitoring was encouraged through the maintenance of a diet diary, which was developed and provided at the initial assessment. The telephone calls were conducted according to a structured topic guide. The intervention group also received structured educational materials developed specifically for the Emirati community. In all of the patients, all of the outcome measures were assessed at baseline, 3 months and 6 months

the patients attempted to adhere to a healthy lifestyle. The intervention group also received structured educational materials developed specifically for the Emirati community regarding sources of carbohydrates and dietary fibre, physical activity, eating out, and healthy cooking methods. The patients in the control group received standard care i.e., 3 individual sessions scheduled at baseline, 1 month and 6 months. These patients received general dietary guideline handouts and calorie-based diets, were provided goals for lifestyle habits and were encouraged to employ self-monitoring. However, the control group was not supported with goal charts, diet diaries or structured nutrition education. All of the consultations for the intervention were delivered within the time allocated to meet the dietitian (Fig. 2).

\section{Outcome measures}

The trial was designed to be integrated into usual clinical practice; therefore, all of the outcomes measures were regular tests or measurements performed during routine assessments and were abstracted from the patients' records. The trial's primary outcome measure was HbA1c level. Secondary outcomes were weight, BMI, body composition analysis, lipid profile (serum cholesterol, low-density lipoprotein cholesterol (LDL-C), high-density lipoprotein cholesterol (HDL-C), and triglycerides (TG)), blood pressure, carbohydrate intake in grams, minutes of moderate physical exercise and medications for type 2 diabetes. The HbA1c and lipid profile (LDL, HDL, triglycerides, and total cholesterol) data were obtained using a Roche COBAS 6000 auto analyser (Mannheim, Germany). Systolic and diastolic blood pressures were measured in the right arm while the patient was seated and after he/she had rested for 10 minutes. The medications used by the patients were abstracted from their electronic records and included all oral anti-diabetic agents as well as insulin. Weight and height were measured using a SECA electronic scale (Hamburg, Germany). Body composition analysis (BCA), presented as fat mass $(\mathrm{Kg})$, muscle mass $(\mathrm{Kg})$ and water mass $(\mathrm{Kg})$, was based on bioelectrical impedance using the InBody-230 analyser (Biospace, Dogok-dong, South Korea) under standard operating procedures. Carbohydrate intake was measured from the patients' three-day food records using the American Dietetic Association food exchange system [22]. These values were then converted into grams of carbohydrates. The carbohydrate exchanges included cereals, fruits, dairy products, vegetables, legumes and other carbohydrates. Physical exercise, was self-reported as number of minutes per week of physical exercise with moderate intensity and was then 
averaged per day. Patients were given examples of moderate physical exercise commonly practiced by the Emirati community. Metabolic measures were performed as part of routine management in the diabetes clinic. The anthropometric, dietary and physical activity measures were assessed by the dietitian as part of the standard care assessments in the lifestyle clinic. For study participants, all of the measures were assessed at baseline, 3 months and 6 months. All of the enrolled participants were invited to attend a follow-up visit at 1 year.

\section{Ethics, consent and permissions}

This study was approved by the UAE Ministry of Health research ethics committee (reference number 032013-07). Additionally, all of the patients provided informed consent to participate in this study.

\section{Statistical analysis}

The sample size was calculated based on the assumption of observing a $10 \%$ reduction in the mean HbA1c level, the primary outcome measure, at 6 months post-intervention. For a power of $80 \%$, the required sample size was estimated to be 50 patients. The data were presented as the mean \pm standard deviation. The group means were compared at baseline using Student's t-test, and one-way ANOVA was performed to compare the means between the groups at different time points. Bonferroni correction was used for all post hoc analyses to control for family-wise error; however, if the initial ANOVA was not significant, no further analysis was conducted. All of the tests were performed two-sided at a significance level of $P \leq 0.05$. The data analysis was performed in accordance with the intention-to-treat analysis protocol.

\section{Results}

The baseline characteristics of the subjects in the intervention and control groups are presented in Table 1. Females composed almost $65 \%$ of the intervention and control groups. There was no significant difference between the intervention and the control groups in HbA1c level, the primary outcome measure, at baseline. On the other hand, the two groups were significantly different in systolic blood pressure $(P=0.01)$ and diastolic blood pressure $(\mathrm{p}=0.00)$ at baseline. Also the intervention group had higher weight than the control group, although non-significant $(\mathrm{p}=0.06)$.

\section{$\mathrm{HbA1c}$, obesity measures and cardiovascular disease risk (CVD) factors}

Changes in HbA1c levels, weight, body composition and CVD risk factors are shown in Table 2. HbA1c levels, the primary outcome measure of the study, changed significantly in the intervention group at three and six months compared to baseline. No significant change was
Table 1 Baseline patient characteristics

\begin{tabular}{llll}
\hline & Intervention $^{\mathrm{a}}$ & Control $^{\mathrm{b}}$ & $P$ value \\
\hline N (Females) & $18(12)$ & $17(11)$ & \\
Age (Years) & $38.37 \pm 9.08$ & $42.53 \pm 10.9$ & 0.87 \\
Duration of diabetes (Years) & $6.75 \pm 7.59$ & $6.8 \pm 6.33$ & 0.98 \\
HbA1c (\%) & $9.39 \pm 1.55$ & $9.11 \pm 1.37$ & 0.46 \\
Obesity measures & & & \\
$\quad$ Weight (Kg) & $94.48 \pm 16.81$ & $84.41 \pm 12.7$ & 0.06 \\
Height (m) & $1.63 \pm 0.07$ & $1.59 \pm 0.087$ & 0.11 \\
BMI (Kg/m ${ }^{2}$ ) & $35.87 \pm 5.67$ & $33.89 \pm 6.54$ & 0.35 \\
Body composition & & & \\
$\quad$ Fat mass (Kg) & $41.93 \pm 13.18$ & $36.76 \pm 11.29$ & 0.23 \\
$\quad$ Muscle mass (Kg) & $14.04 \pm 2.07$ & $12.41 \pm 1.88$ & $0.03^{\mathrm{b}}$ \\
$\quad$ Water mass (Kg) & $38.88 \pm 5.67$ & $34.24 \pm 4.29$ & $0.01^{\mathrm{b}}$ \\
CVD risk factors & & & \\
HDL cholesterol (mmol/L) & $1.05 \pm 0.56$ & $1.21 \pm 0.37$ & 0.1 \\
LDL cholesterol (mmol/L) & $3.11 \pm 1.05$ & $2.94 \pm 0.77$ & 0.86 \\
Total cholesterol (mmol/L) & $4.77 \pm 1.05$ & $4.59 \pm 0.81$ & 0.93 \\
Triglycerides (mmol/L) & $2.58 \pm 1.33$ & $2.1 \pm 1.9$ & 0.37 \\
Systolic blood pressure ( $\mathrm{mmHg})$ & $136 \pm 12$ & $122 \pm 14$ & $0.01^{\mathrm{b}}$ \\
Diastolic blood pressure (mmHg) & $76 \pm 8$ & $67 \pm 9$ & $0.00^{\mathrm{b}}$ \\
\hline Con & & & \\
\hline
\end{tabular}

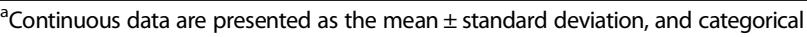
data are presented as frequencies

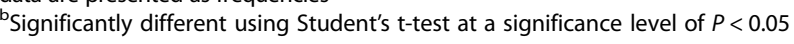

observed in the control group for the same periods, as illustrated in Fig. 3. The patients who had diabetes for less than 5 years $(n=10)$ exhibited significantly greater improvement in HbA1c levels at 3 months $(-2.44 \pm 1.89)$ than the patients with a disease duration of more than 5 years $(\mathrm{n}=8,-0.46 \pm 0.95)(P=0.01)$. The reduction in HbA1c levels remained larger at 6 months between those with a diabetes duration more than and less than 5 years, although the difference was not statistically significant $(-1.77 \pm-0.43$ vs. $-0.43 \pm 1.09)$. These findings were not observed in the control group. Both males and females exhibited a significant reduction in HbA1c levels at 3 months; however, the females did not maintain the significant change at 6 months. Additionally, for both groups, there were no significant changes in the doses of any of the oral anti-diabetic agents or in insulin at any of the time points.

The mean weight of the subjects in both the intervention and control groups did not change significantly at the end of the study $(P>0.05)$, as shown in Table 2 . Similarly, the body composition, i.e., fat mass and muscle mass, of the subjects did not change significantly $(P>0.05)$, with the exception of water mass, which was significantly reduced at 6-month follow-up in the control group $(P=0.03)$. Additionally, no significant changes in lipid profiles or diastolic and systolic blood pressures 
Table 2 Changes in HbA1C (\%), obesity measures and CVD risk factors at 3 months and 6 months

\begin{tabular}{|c|c|c|c|c|c|c|}
\hline & \multicolumn{3}{|c|}{ Intervention $(n=18)^{a}$} & \multicolumn{3}{|l|}{ Control $(n=17)^{a}$} \\
\hline & 3 months & 6 months & $P$ value $^{b}$ & 3 months & 6 months & $P$ value $^{b}$ \\
\hline HbA1c (\%) & $(-) 1.56 \pm 1.81^{c}$ & $(-) 1.17 \pm 2.11^{c}$ & 0.00 & $0.1 \pm 1.37$ & $(-) 0.17 \pm 1.79$ & 0.74 \\
\hline \multicolumn{7}{|l|}{ Obesity measures } \\
\hline Weight (Kg) & $(-) 0.29 \pm 3.12$ & $(-) 0.72 \pm 4.3$ & 0.65 & $(-) 0.25 \pm 1.22$ & $(-) 0.41 \pm 2.56$ & 0.76 \\
\hline $\mathrm{BMI}\left(\mathrm{Kg} / \mathrm{m}^{2}\right)$ & $(-) 0.01 \pm 1.1$ & $0.14 \pm 1.41$ & 0.97 & $(-) 0.05 \pm 0.54$ & $0.14 \pm 1.44$ & 0.97 \\
\hline \multicolumn{7}{|l|}{ Body composition } \\
\hline Fat mass (Kg) & $0.00 \pm 2.56$ & $(-) 0.08 \pm 3.73$ & 0.98 & $(-) 1.21 \pm 3.23$ & $(-) 1.19 \pm 3.91$ & 0.26 \\
\hline Muscle mass (Kg) & $(-) 0.09 \pm 0.46$ & $(-) 0.15 \pm 0.49$ & 0.35 & $0.27 \pm 0.93$ & $0.17 \pm 0.83$ & 0.49 \\
\hline Water mass (Kg) & $(-) 0.14 \pm 1.2$ & $(-) 2.07 \pm 7.17$ & 0.34 & $0.99 \pm 1.67$ & $0.79 \pm 1.36$ & 0.03 \\
\hline \multicolumn{7}{|l|}{ CVD risk factors } \\
\hline $\mathrm{HDL}(\mathrm{mmol} / \mathrm{L})$ & $0.00 \pm 0.13$ & $(-) 0.01 \pm 0.15$ & 0.96 & $(-) 0.01 \pm 0.17$ & $(-) 0.01 \pm 0.19$ & 0.96 \\
\hline $\mathrm{LDL}(\mathrm{mmol} / \mathrm{L})$ & $(-) 0.08 \pm 0.89$ & $(-) 0.09 \pm 0.95$ & 0.86 & $(-) 0.17 \pm 0.6$ & $(-) 0.24 \pm 0.77$ & 0.21 \\
\hline Total cholesterol (mmol/dL) & $0.17 \pm 0.69$ & $(-) 0.13 \pm 0.83$ & 0.58 & $(-) 0.18 \pm 0.63$ & $(-) 0.14 \pm 0.94$ & 0.38 \\
\hline Triglycerides (mmol/dL) & $(-) 0.37 \pm 1.05$ & $(-) 0.18 \pm 0.76$ & 0.14 & $(-) 0.09 \pm 0.4$ & $0.12 \pm 0.78$ & 0.43 \\
\hline Systolic blood pressure ( mmHg) & $(-) 3.18 \pm 11.67$ & $(-) 1.71 \pm 12.07$ & 0.47 & $0.25 \pm 13.16$ & $3.13 \pm 15.12$ & 0.44 \\
\hline Diastolic blood pressure $(\mathrm{mmHg})$ & $(-) 0.18 \pm 8.9$ & $0.76 \pm 7.48$ & 0.89 & $2.50 \pm 5.35$ & $4.19 \pm 8.48$ & 0.12 \\
\hline
\end{tabular}

${ }^{\mathrm{a}}$ Data are presented as the mean \pm standard deviation

${ }^{b}$ One-way ANOVA was used for between-groups comparisons, with a significance level of $P<0.05$

${ }^{\mathrm{c}} P$ value $<0.0167$ after Bonferroni correction

were observed in the intervention and control groups at the end of the study $(P>0.05)$. However, at 6 months, $41 \%$ of the patients in the intervention group met the American Diabetes Association targets for LDL cholesterol $(<2.6 \mathrm{mmol} / \mathrm{L})$, compared to $24 \%$ at baseline, whereas only $31 \%$ of the patients in the control group met the targets at 6 months compared to $19 \%$ at baseline.

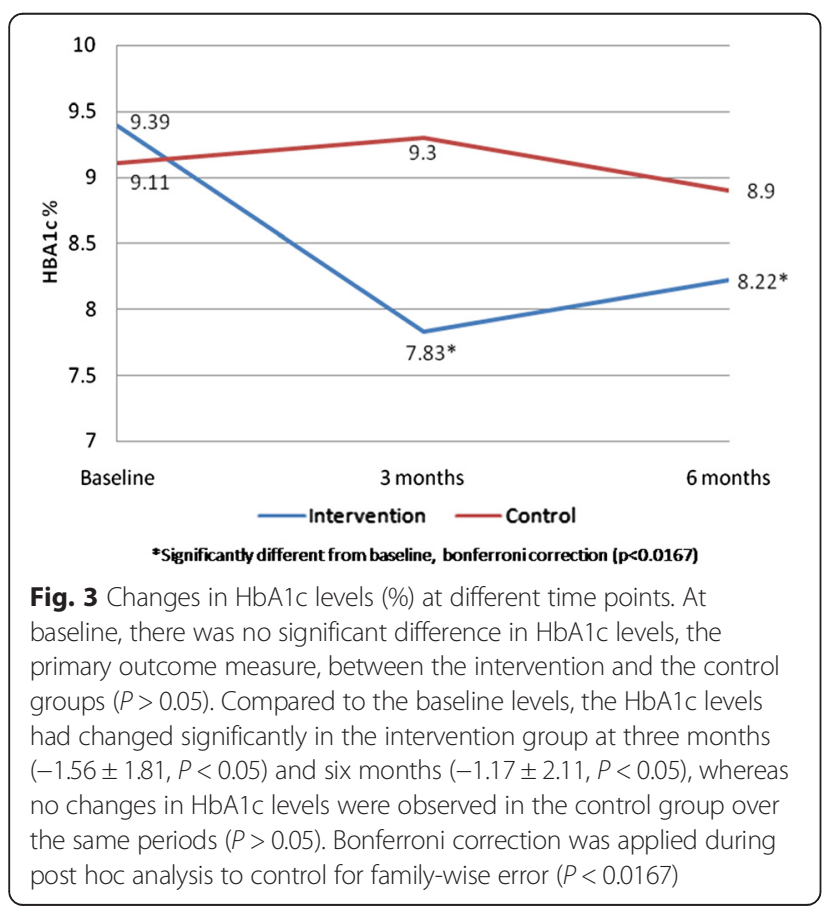

\section{Carbohydrate intake and physical exercise}

Table 3 shows the patients' carbohydrate intake (in grams) from different sources and their physical exercise throughout the study. At baseline, the intervention and control groups did not differ with respect to the intake of carbohydrates from all sources i.e. cereals, fruits, dairy products, vegetables, legumes and other carbohydrates like sweets $(P>0.05)$. Physical exercise was significantly higher in the control group than the intervention group $(\mathrm{p}<0.05)$, at baseline. In the intervention group, the intake of carbohydrates from cereals was significantly reduced at 3 and 6 months $(P<0.01)$. In contrast, there was an increase in the intake from this source in the control group at 6 months, although the increase was not statistically significant $(P>0.05)$ (Fig. 4). There was a significant reduction in the intake from dairy products in the intervention group at 3 months $(\mathrm{p}<0.05)$, however, the intake increased to values close to baseline at 6 months. Similarly, total carbohydrate intake decreased significantly in the intervention group $(P<0.05)$ at 3 months and had maintained its significant reduction at 6 months $(P<0.05)$. There was a non-significant increase in the level of physical exercise ( $\mathrm{min} /$ day) in both the groups at 3 months, as displayed in Table 3. This increase was maintained by the intervention group at 6 months, whereas the levels had reduced in the control group.

\section{1 year follow-up}

At the 1-year follow-up, the effect of the intervention on HbA1c, the primary outcome, remained significant 
Table 3 Dietary intake of carbohydrates and physical exercise at baseline, 3 months and 6 months

\begin{tabular}{|c|c|c|c|c|c|c|c|c|}
\hline \multirow[b]{2}{*}{ Carbohydrate intake (g/day) } & \multicolumn{4}{|c|}{ Intervention $(n=18)^{a}$} & \multicolumn{4}{|l|}{ Control $(n=17)^{a}$} \\
\hline & Baseline & 3 months & 6 months & $P$ value $^{\mathrm{b}}$ & Baseline & 3 months & 6 months & $P$ value ${ }^{\mathrm{b}}$ \\
\hline Cereals & $172.92 \pm 73.8$ & $130.42 \pm 55.12^{c}$ & $140.00 \pm 70.17^{c}$ & 0.00 & $149.5 \pm 81.49$ & $145.00 \pm 63.56$ & $164 \pm 58.60$ & 0.54 \\
\hline Fruits & $15.00 \pm 17.06$ & $10.83 \pm 14.37$ & $23.33 \pm 23.84$ & 0.03 & $41.00 \pm 28.57$ & $37.5 \pm 35.6$ & $40.5 \pm 35.05$ & 0.91 \\
\hline Dairy products & $10.00 \pm 7.97$ & $3.67 \pm 4.67^{\mathrm{a}}$ & $8.67 \pm 8.54$ & 0.00 & $10.00 \pm 9.21$ & $6.40 \pm 6.74$ & $8.00 \pm 8.94$ & 0.22 \\
\hline Vegetables & $6.94 \pm 3.04$ & $6.67 \pm 5.42$ & $6.67 \pm 4.20$ & 0.96 & $4.67 \pm 4.99$ & $3.67 \pm 2.87$ & $7.00 \pm 4.76$ & 0.09 \\
\hline Legumes & $3.75 \pm 6.43$ & $2.08 \pm 5.02$ & $2.08 \pm 5.02$ & 0.52 & $4.00 \pm 8.60$ & $1.50 \pm 4.06$ & $2.00 \pm 5.01$ & 0.38 \\
\hline Other carbohydrates & $10.83 \pm 10.09$ & $16.67 \pm 23.51$ & $18.75 \pm 23.89$ & 0.47 & $7.00 \pm 14.35$ & $0.57 \pm 0.95$ & $8.00 \pm 14.35$ & 0.95 \\
\hline Total carbohydrates & $219.44 \pm 84.18$ & $170.33 \pm 75.76^{\mathrm{a}}$ & $199.5 \pm 86.28^{a}$ & 0.00 & $216.17 \pm 89.14$ & $202.55 \pm 90.3$ & $237.87 \pm 59.42$ & 0.3 \\
\hline Physical exercise (min/day) & $4.67 \pm 9.89$ & $9.12 \pm 17.39$ & $12.17 \pm 18.60$ & 0.11 & $10.00 \pm 15.99$ & $15.19 \pm 22.02$ & $12.31 \pm 13.57$ & 0.68 \\
\hline
\end{tabular}

${ }^{\mathrm{a}}$ Data are presented as the mean \pm standard deviation

${ }^{\mathrm{b}}$ One-way ANOVA was used for between-groups comparisons, with a significance level of $P<0.05$

${ }^{c} P$ value $<0.0167$ after Bonferroni correction

$(\mathrm{p}<0.05)$ with an average of $8.27 \pm 1.99 \%$ compared to the baseline level $9.39 \pm 1.55 \%$. In contrast, no significant change between baseline $(9.11 \pm 1.37 \%)$ and the 1-year follow-up $(8.7 \pm 1.37 \%)$ was observed in the control group. Additionally, there were no significant changes in the other secondary outcomes in either the control or the intervention group, with the exception of diastolic blood pressure, which in the control group, was significantly higher at 1 year $(72.0 \pm 8.0)$ compared to baseline $(67.0 \pm 9.0)$.

\section{Discussion}

The goal of this study was to develop a translational behavioural lifestyle programme and to assess its effectiveness in improving glycaemic control in Emirati patients with type 2 diabetes compared to standard care practices. We found that the intervention effectively achieved a significant reduction in HbA1c levels over a period of six months, whereas no significant change was observed in the control group during this period. The

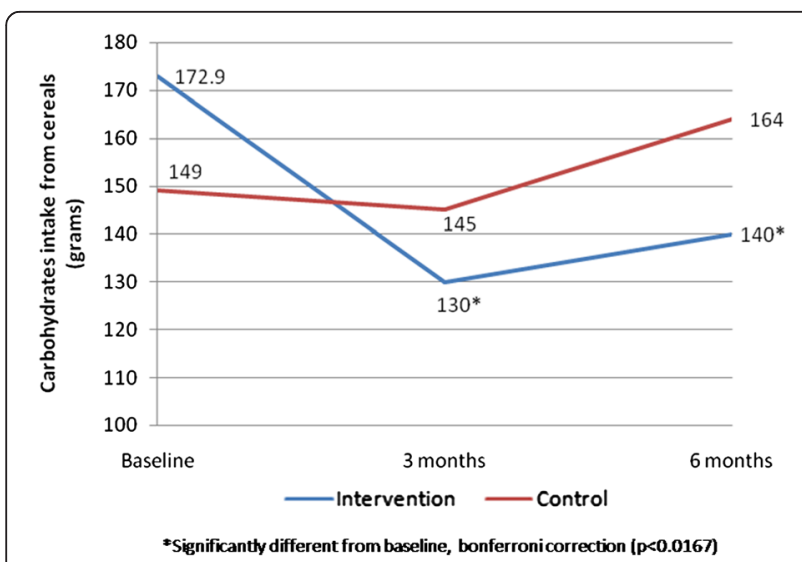

Fig. 4 Changes in carbohydrate intake from cereals at different time points. *Significantly different from baseline, Bonferroni correction $(P<0.0167)$ patients in the intervention group also maintained this reduction 1 year after completing the programme. We also reported a significant reduction in both total carbohydrate intake and carbohydrate intake from cereals over the intervention period in the intervention group, whereas no significant change was observed in the control group.

Our lifestyle intervention used behavioural strategies adapted from CBT. Strong evidence supports the use of CBT as the underpinning theory for lifestyle counselling with the goal of facilitating compliance with behavioural changes [7].

In our study, the two main CBT behavioural strategies used were SMART goal setting and self-monitoring. For instance, all of the patients in the intervention group received individualized SMART goals for carbohydrate intake, which may have facilitated their compliance with this dietary change. Additionally, the patients were instructed to monitor their intake in food journals in an attempt to improve their self-monitoring skills.

Behavioural strategies have been used in large lifestyle intervention studies [8-10] that successfully achieved significant post-intervention improvements in glycaemic control. However, limited numbers of translational behavioural interventions have been performed in type 2 diabetes patients. Of the few existing translational intervention studies, some have shown positive impacts on HbA1c levels at 6 months post-intervention. For instance, Adachi et al. (2013) [18] reported a significant decrease in HbA1c levels at the end of a 6-month behavioural lifestyle programme ( $\mathrm{n}=91,-0.7 \%, P=0.004)$. Similarly, Spencer et al. (2011) [19] reported a decrease of $0.8 \%$ in HbAlc levels post-intervention $(\mathrm{n}=164, P<0.01)$. However, our results revealed slightly larger improvement in the intervention group $(-1.17 \%, P<0.01)$, which could be because half of the patients in the intervention group had diabetes for less than 5 years. However, the duration of diabetes was not reported in the previous studies $[18,19]$. 
An association between improvements in HbA1c levels and the duration of diabetes is well documented in the literature. Reviews have reported that medical nutrition therapy has the greatest effect at the initial diagnosis of diabetes, during which a reduction in HbA1c of 1-2 units is expected [23].

Taking into account the resource constraints encountered in clinical practice, we reduced the number of sessions (8 sessions) included in the intervention to less than the that of the Look Ahead trial, which consisted of 24 sessions in the first 6 months [10]. However, we chose a session number that was greater than the smallest number of sessions associated with successful outcomes, which was 6 sessions [24]. Additionally, four of these sessions were delivered via telephone calls. Telephone calls have been used in many lifestyle interventions because it may reduce the strain on available resources [24]. Telephone calls have also been observed to have an independent positive effect on glycaemic control in type 2 diabetes [25].

On the other hand, one could argue whether the observed weight loss in this study was modest. Our study primary outcome was HbA1c, hence, we chose strategies to improve the glycemic control which are appropriate for the Emirati community like reducing carbohydrates intake [21] which may have negatively affected the achievement of weight loss. Arguably, the contribution of carbohydrates restriction to the calorie deficit may be suggested as insufficient to achieve weight loss compared, for instance, to reducing fat intake.

In regards to weight loss, one could argue that the results observed in our study was modest and whether reducing the number of sessions or restricting carbohydrates was insufficient to achieve. Our study primary outcome was HbA1c, hence, we chose strategies which were believed to improve the glycemic control and is more appropriate to the Emirati community like restricting carbohydrates.

Another lifestyle factor that has proven challenging to improve in real-life settings is physical exercise. Similar to the results of Linmans et al. (2011) [13], who reported no change in exercise levels after a lifestyle intervention applied through primary care, we did not observe a significant increase in physical exercise levels over the intervention period. Similarly, although Adachi et al. (2013) [18] reported a significant reduction in $\mathrm{HbA} 1 \mathrm{c}$ levels 6 months after a lifestyle education programme, the observed change in exercise status was quite modest compared to the change in the targeted dietary habit. These findings indicate that further investigations are needed to identify barriers to physical activity in real life and the key behavioural strategies that can improve compliance to physical activity guidelines.

\section{Strengths and limitations}

One of the main strengths of our study was its translational design-it aimed to test a behavioural lifestyle programme that could be incorporated into standard care practices. Although large, controlled lifestyle trials provide insight into what may work best under ideal situations, reproducing the same results in clinical practice might be challenging This is because in real life, health care professionals work under challenging conditions that act as barriers to successful outcomes. Such barriers include the lack of resources, limited practitioner's time, and demotivated patients [12]; these factors need to be taken into consideration when designing pragmatic trials. In BLIS, we developed a lifestyle programme based on evidence-based behavioural theory and took into account the feasibility of our programme in clinical practice, both in terms of the number of sessions and the intervention delivery method, which future research can build upon. We also developed structured nutrition education materials tailored to the Emirati community, which can be used in communities with the same cultural background.

On the other hand, one of the main limitations of our study was its relatively small sample size despite our efforts to recruit the calculated number of subjects. This was mainly due to patients not coming to their initial assessment with the dietitian for reasons like the time gap between their first visit to the centre and the appointment with the dietitian. Thus, future research may need to overcome such recruitment barriers and to investigate the effectiveness of behavioural lifestyle interventions on a larger scale, possibly utilizing a clusterrandomized controlled design. Additionally, BLIS was conducted in a specialized diabetes centre, and thus the generalizability of our programme to other health care settings, such as hospitals and primary care facilities, remains uncertain.

\section{Conclusion}

In conclusion, this study demonstrated the effectiveness of a translational behavioural lifestyle intervention in improving glycaemic control in Emirati patients with type 2 diabetes. This improvement was evident over the short term and was maintained at 1 year post-intervention. The results of our study add to evidence of the effectiveness of behavioural lifestyle programmes in clinical practice, particularly in the Arab region, which is an area of limited research. More studies with larger sample size are urgently needed to evaluate the long-term effectiveness of behavioural lifestyle programmes. Additionally, future research might need to evaluate the effectiveness of behavioural lifestyle interventions for weight management in clinical practice and to identify the key behavioural strategies that improve compliance to healthy lifestyle practices. 


\section{Abbreviations}

BCA: body composition analysis; BLIS: behavioural lifestyle intervention study; BMI: body mass index; CBT: cognitive behavioural theory; CVD: cardiovascular disease; DPP: diabetes prevention program; IDF: International Diabetes Federation; UAE: United Arab Emirates; RCDR: Rashid Centre for Diabetes and Research; SMART: specific, measurable, achievable, relevant and timed; LDL-c: low-density lipoprotein cholesterol; HDL-c: high-density lipoprotein cholesterol; TG: triglycerides.

\section{Competing interests}

All of the authors confirm that they have no competing interests.

\section{Author' contributions}

SA, AS and SA (3) participated in the design of the study. SA, AS, SA (2), and SV conducted the research. SA and AS analysed the data. SA and AS wrote the paper. SA and SA (3) were responsible for overall supervision and had primary responsibility for the final outcome. All of the authors read and approved the final manuscript.

\section{Acknowledgements}

The authors would like to acknowledge the Global Health Partner Diabetes Centre UAE for providing the funds required to conduct the research and for continuous support throughout the process.

\section{Author details}

${ }^{1}$ Clinical Dietitian, Rashid Center for Diabetes and Research, Ministry of Health, P.O BOX 21499, Ajman, United Arab Emirates. ${ }^{2}$ Diabetes Clinic, Rashid Center for Diabetes and Research, Ministry of Health, Ajman, United Arab Emirates.

Received: 9 July 2015 Accepted: 28 October 2015 Published online: 19 November 2015

\section{References}

1. World health organization regional office for the Eastern Mediterranean. Country cooperation strategy for WHO and United Arab Emirates 2005-2009. Cairo: EMRO; 2006.

2. International Diabetes Federation. IDF Diabetes Atlas update poster, $6^{\text {th }}$ edn. Brussels, Belguim: International Diabetes Federation; 2014.

3. Al-Maskari F, El-Sadig M, Nagelkerke N. Assessment of the direct medical costs of diabetes mellitus and its complications in the United Arab Emirates. BMC Public Health. 2010;10:679.

4. American Diabetes Association. Standards of medical care in diabetesd2014. Diabetes Care. 2014;37 Suppl 1:S14-80.

5. Al-Kaabi J, Al-Maskari F, Saadi H, Afandi B, Parkar H, Nagelkerke N. Assessment of dietary practice among diabetic patients in the United Arab Emirates. The review of diabetic studies: RDS. 2008;5(2):110.

6. Al-Kaabi J, Al-Maskari F, Saadi H, Afandi B, Parkar H, Nagelkerke N. Physical activity and reported barriers to activity among type 2 diabetic patients in the United Arab Emirates. The review of diabetic studies: RDS. 2009;6(4):271.

7. Spahn JM, Reeves RS, Keim KS, Laquarta I, Kellogg M, Jortberg B, et al. State of the evidence regarding behavior change theories and strategies in nutrition counseling to facilitate health and food behavior change. J Am Diet Assoc. 2010;110(6):879-91.

8. Group DPPR. Reduction in the incidence of type 2 diabetes with lifestyle intervention or metformin. N Engl J Med. 2002;346(6):393.

9. Unick JL, Beavers D, Jakicic JM, Kitabchi AE, Knowler WC, Wadden TA, et al. Effectiveness of lifestyle interventions for individuals with severe obesity and type 2 diabetes results from the Look AHEAD trial. Diabetes Care. 2011;34(10):2152-7.

10. Delahanty LM, Nathan DM. Implications of the Diabetes Prevention Program and Look AHEAD clinical trials for lifestyle interventions. J Am Diet Assoc. 2008;108(4):S66-72.

11. Dunkley AJ, Bodicoat DH, Greaves CJ, Russell C, Yates T, Davies MJ, et al. Diabetes prevention in the real world: effectiveness of pragmatic lifestyle interventions for the prevention of type 2 diabetes and of the impact of adherence to guideline recommendations: a systematic review and metaanalysis. Diabetes Care. 2014;37(4):922-33.

12. Cardona-Morrell M, Rychetnik L, Morrell SL, Espinel PT, Bauman A. Reduction of diabetes risk in routine clinical practice: are physical activity and nutrition interventions feasible and are the outcomes from reference trials replicable? A systematic review and meta-analysis. BMC Public Health. 2010;10:653.

13. Linmans JJ, Spigt MG, Deneer L, Lucas AE, de Bakker M, Gidding LG, et al. Effect of lifestyle intervention for people with diabetes or prediabetes in real-world primary care: propensity score analysis. BMC Fam Pract. 2011;12(1):95.

14. Lakerveld J, Bot SD, Chinapaw MJ, van Tulder MW, Kostense PJ, Dekker JM, et al. Motivational interviewing and problem solving treatment to reduce type 2 diabetes and cardiovascular disease risk in real life: a randomized controlled trial. Int J Behav Nutr Phys Act. 2013;10(47):10.1186.

15. Barclay C, Procter KL, Glendenning R, Marsh P, Freeman J, Mathers N. Can type 2 diabetes be prevented in UK general practice? A lifestyle-change feasibility study (ISAIAH). British Journal of General Practice. 2008;58(553):541-7.

16. Ali MK, Echouffo-Tcheugui JB, Williamson DF. How effective were lifestyle interventions in real-world settings that were modeled on the Diabetes Prevention Program? Health Aff. 2012;31(1):67-75.

17. Schellenberg ES, Dryden DM, Vandermeer B, Ha C, Korownyk C. Lifestyle interventions for patients with and at risk for type 2 diabetes: a systematic review and meta-analysis. Ann Intern Med. 2013;159(8):543-51.

18. Adachi M, Yamaoka K, Watanabe M, Nishikawa M, Kobayashi I, Hida E, et al. Effects of lifestyle education program for type 2 diabetes patients in clinics: a cluster randomized controlled trial. BMC Public Health. 2013;13(1):467.

19. Spencer MS, Rosland AM, Kieffer EC, Sinco BR, Valerio M, Palmisano G, et al. Effectiveness of a community health worker intervention among African American and Latino adults with type 2 diabetes: a randomized controlled trial. Am J Public Health. 2011;101(12):2253-60.

20. Welschen LM, van Oppen P, Bot SD, Kostense PJ, Dekker JM, Nijpels G. Effects of a cognitive behavioural treatment in patients with type 2 diabetes when added to managed care; a randomised controlled trial. J Behav Med. 2013;36(6):556-66.

21. Evert AB, Boucher JL, Cypress M, Dunbar SA, Franz MJ, Mayer-Davis EJ, et al. Nutrition therapy recommendations for the management of adults with diabetes. Diabetes Care. 2013;36(11):3821-42.

22. Association, A.D. and A.D. Association, Choose Your Foods: Exchange Lists for Weight Management. 2008. American Diabetes Association, Alexandria, VA and American Diabetes Association, Chicago, IL.

23. Pastors JG, Franz MJ, Warshaw H, Daly A, Arnold MS. How effective is medical nutrition therapy in diabetes care? J Am Diet Assoc. 2003;103(7):827-31.

24. Baker MK, Simpson K, Lloyd B, Bauman AE, Singh MA. Behavioral strategies in diabetes prevention programs: a systematic review of randomized controlled trials. Diabetes Res Clin Pract. 2011;91(1):1-12.

25. Liang X, Wang Q, Yang X, Cao J, Chen J, Mo X, et al. Effect of mobile phone intervention for diabetes on glycaemic control: a meta-analysis. Diabet Med. 2011;28(4):455-63.

26. Greaves CJ et al. Motivational interviewing for modifying diabetes risk: a randomised controlled trial. British J Gen Prac. 2008;58(553):535-40.

\section{Submit your next manuscript to BioMed Central and take full advantage of:}

- Convenient online submission

- Thorough peer review

- No space constraints or color figure charges

- Immediate publication on acceptance

- Inclusion in PubMed, CAS, Scopus and Google Scholar

- Research which is freely available for redistribution 